\title{
Defect Physics in Photovoltaic Materials Revealed by Combined High-Resolution Microscopy and Density-Functional Theory Calculation
}

\author{
Yanfa Yan ${ }^{1}$, Chen $\mathrm{Li}^{2}$, Yelong Yu ${ }^{1}$, Wan-Jian Yin ${ }^{1}$, Zhiwei Wang ${ }^{1}$, Naba R. Paudel ${ }^{1}$, Jonathan \\ Poplawsky ${ }^{2}$, Timothy J. Pennycook ${ }^{2}$, Wyatt K. Metzger ${ }^{3}$, Ingrid Repins ${ }^{3}$, Mowafak M. Al-Jassim ${ }^{3}$, \\ Stephen J. Pennycook ${ }^{4}$ \\ ${ }^{1}$ Department of Physics and Astronomy, The University of Toledo, Toledo, OH, USA \\ ${ }^{2}$ Materials Science and Technology Division, Oak Ridge National Laboratory, Oak Ridge, TN, USA \\ ${ }^{3}$ National Renewable Energy Laboratory, Golden, CO, USA \\ ${ }^{4}$ Department of Materials Science and Engineering, University of Tennessee, Knoxville, TN, USA
}

Thin-film solar cells based on polycrystalline $\mathrm{CdTe}, \mathrm{Cu}(\mathrm{In}, \mathrm{Ga}) \mathrm{Se}_{2}$ (CIGS), and $\mathrm{Cu}_{2} \mathrm{ZnSnSe}_{4}$ (CZTSe) have demonstrated high solar-to-electricity conversion efficiencies [1]. Typically, polycrystalline thinfilm solar cells are expected to exhibit poor performance compared to their single-crystalline counterparts, due to the existence of unavoidable structural defects such as dislocations and grain boundaries (GBs). It is surprising, therefore, that CdTe-, CIGS-, and CZTSe-based polycrystalline thinfilm solar cells have achieved higher efficiencies than their single-crystalline counterparts.

Here, we present our results on the study of the atomic structure and electronic properties of structural defects including stacking faults, twins, dislocations, and GBs in CdTe, CIGS, and CZTSe using a combination of aberration-corrected scanning transmission electron microscopy and first-principles density-functional theory calculation. Polycrystalline CdTe thin films were grown by closed-space sublimation, whereas CIGS and CZTSe thin films were deposited by co-evaporation. Atomic resolution scanning transmission electron microscopy (STEM) images were acquired with Nion UltraSTEM 100 and 200 microscopes. DFT calculations used the local density approximation with the Hubbard U correction. We found that intrinsic GBs in CdTe, CIGS, and CZTSe create deep levels mainly due to the anion-anion "wrong" bonding. However, these deep levels can be removed through extrinsic and/or intrinsic passivation. Special intra-grain partial dislocation pairs can even help separate photo-generated carriers and adjust recombination.

Figure 1 shows the atomic configuration of several partial dislocations observed within CdTe grains. As Te is slightly heavier than $\mathrm{Cd}$, we are able to distinguish them from their image intensities [2]. The small blue and large yellow circles indicate $\mathrm{Cd}$ and Te columns. The red box in Fig. 1(a) indicates an intrinsic stacking fault, while the red boxes in (b) and (c) indicate extrinsic stacking faults. Figure 2 (a) and (b) show the supercells containing the observed $\mathrm{Cd}$ and Te cores. The calculated band structures and density of states (DOSs) in Fig. 2(c) and (d) show how the Cd core acts as a shallow donor and the Te core acts as a shallow acceptor. When these two cores form a partial dislocation pair, the $\mathrm{Cd}$ core will transfer one electron to the Te core. As shown in Fig. 2 (e) and (f), the charge transfer causes band bending, which helps separate photo-generated carriers and avoids their recombination [3]. However, GBs exhibit much different behavior. Figure 3(a) and (b) show the Z-contrast image of a $\Sigma 3(112) \mathrm{GB}$ in CdTe and the derived atomic structure, respectively. This GB contains strong Te-Te "wrong" bonding, which leads to deep defect states in the band gap of CdTe and therefore is detrimental for carrier separation and collection (Fig. 3(c)). However, Cl segregation at the GB can fully passivate the defect states. In CIGS and CZTSe, intrinsic GBs are also found to create deep defect states, which can also be passivated. 


\section{References:}

[1] M. A. Green, K. Emery, Y. Hishikawa, W. Warta, and E. D. Dunlop, Prog. Photovolt: Res. Appl. 21 (2013), p. 827.

[2] C. Li, J. Poplawsky, Y. Wu, A. R. Lupini, A. Mouti, D. N. Leonard, N. Paudel, K. Jones, W. Yin, M. Al-Jassim, Y. Yan and S. J. Pennycook, Ultramicroscopy 134 (2013), p.113.

[3] C. Li, Y. Wu, T. J. Pennycook, A. R. Lupini, D. N. Leonard, W. Yin, N. Paudel, M. Al-Jassim, Y. Yan and S. J. Pennycook, Phys. Rev. Lett. 111 (2013), p.096403.
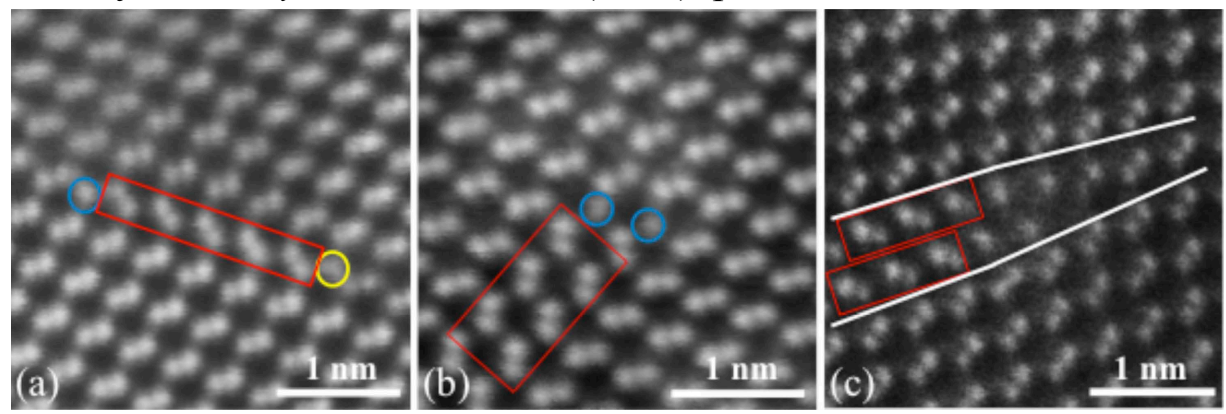

Figure 1. Z-contrast images show the partial dislocations at the ends of stacking faults. (a) Shockley partial dislocation pair: A single $\mathrm{Cd}$ and Te column at each end of an intrinsic stacking fault. (b) Two unpaired Cd columns in a Shockley partial dislocation at the end of an extrinsic stacking fault. (c) A Frank partial dislocation at the end of an extrinsic stacking fault.

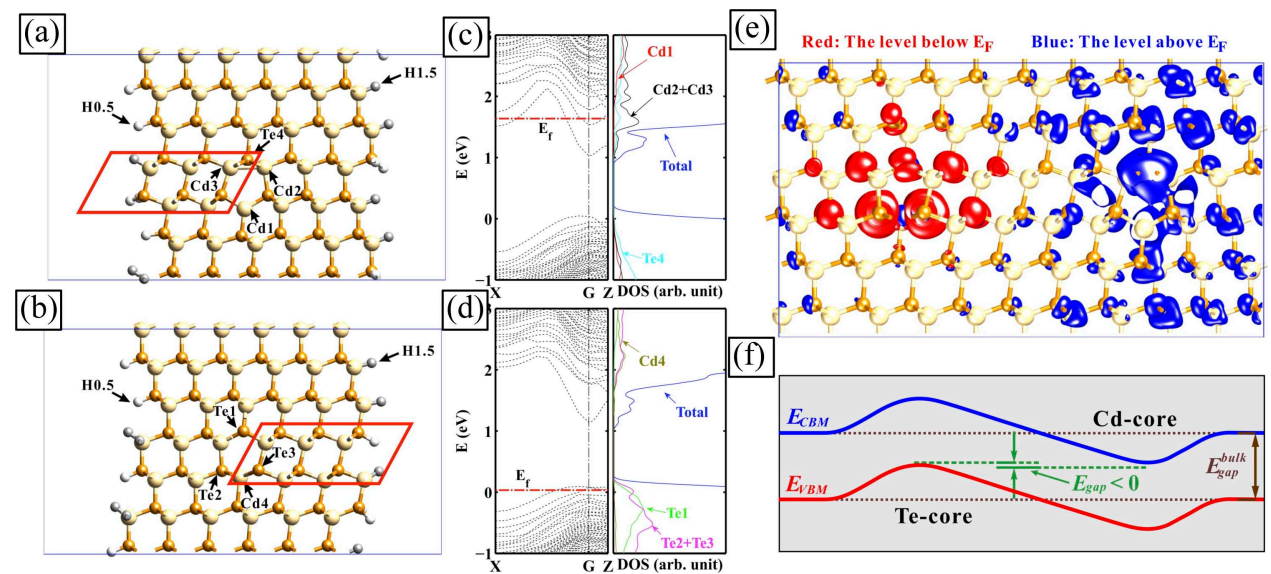

Figure 2. Supercells containing (a) a Cd core and (b) a Te core of an extrinsic stacking fault. Band structure and DOS of individual cores: (c) Cd-core; (d) Te-core. (e) Calculated charge density maps associated with the gap states just above and just below the Fermi level marked in Fig. 2(c). (b) Schematic showing the band bending caused by the charge transfer from the Te core to the Cd core.
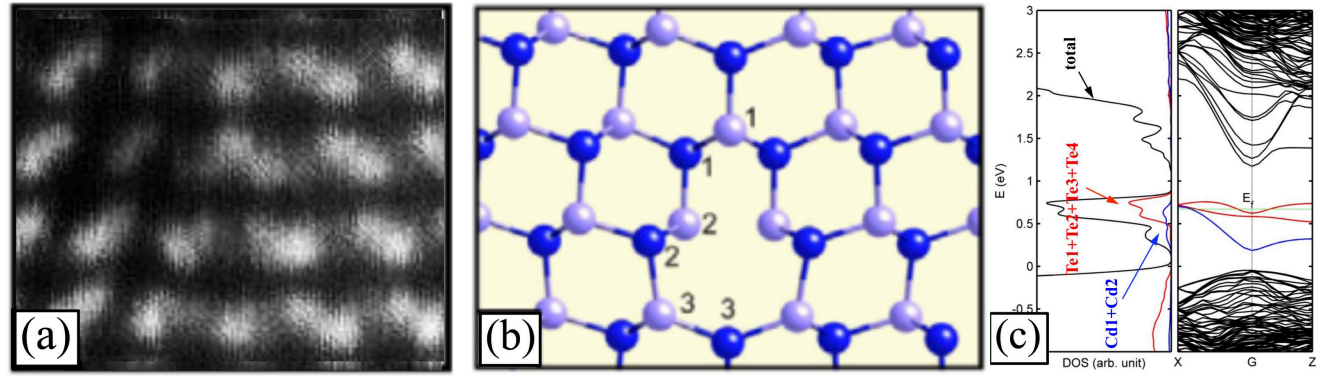

Figure 3. (a) Z-contrast image of a $\Sigma 3(112) \mathrm{GB}$ in CdTe; (b) Atomic-structure derived from the Z-contrast image; (3) Calculated electronic properties of the GB seen in Fig. 3(a). 